\title{
MicroRNA-145 Aggravates Hypoxia- Induced Injury by Targeting Rac1 in H9c2 Cells
}

Ximing Wang ${ }^{\mathrm{a}}$ Yanxia Zhang ${ }^{\mathrm{b}}$ Hongshan Wang ${ }^{\mathrm{a}}$ Genshang Zhao ${ }^{\mathrm{a}}$ Xianen Fa aDepartment of Cardiovascular Surgery, The Second Affiliated Hospital of Zhengzhou University, Zhengzhou, 'bSterilization and Supply Center, The Second Affiliated Hospital of Zhengzhou University, Zhengzhou, China 